\title{
Preparation and Application of Graphene Modified Heated Glassy Carbon Electrode
}

\author{
Hao Cheng \\ College of Biotechnology and Food Engineering, Hefei \\ University of Technology, Hefei, China,230009 \\ Department of Biological and Chemical Engineering, \\ Guangxi University of Science and \\ Technology,Liuzhou,China,545006 \\ Guangxi Key Laboratory of Green Processing of Sugar \\ Resources, Guangxi University of Science and \\ Technology,Liuzhou,China,545006 \\ Key Laboratory for Processing of Sugar Resources of \\ Guangxi Higher Education Institutes, Guangxi University of \\ Science and Technology,Liuzhou,China,545006
}

\begin{abstract}
In this paper, the graphene is prepared by chemical oxidation and chemical reduction, the finished grapheme is modified on the surface of heated glassy carbon electrode through drop casting, the graphene modified heated glassy carbon electrode(GR/HGCE) is then prepared, the characteristics of graphene material and analytical \& detective techniques is combined, thus increasing the effective surface area and surface properties of heated glassy carbon electrode, the homemade graphene is characterized by $\mathrm{X}$-ray powder diffraction, infrared ray, scanning electron microscopy etc. and the GR / HGCE is characterized by cyclic voltammetry (CV). The electrochemical activity of ACOP is studied based on GR /HGCE, and the new approach that adopts GR/HGCE to detect ACOP is formed.
\end{abstract}

Keywords-Graphene; Heated Electrode; Glassy Carbon Electrode; Acetaminophen

\section{INTRODUCTION}

Graphene (Graphene, GR) is a new type of two-dimensional plane carbon nanomaterial discovered in 2004, its special single atomic layer structure determines its unique structure and excellent performance, it posses good conductivity, higher special surface, good mechanical stability, chemical stability and thermal stability and has been widely used in electrical chemistry and electroanalytical chemistry in recent years ${ }^{[1-2]}$.

Acetaminophen (ACOP) ${ }^{[3]}$ is acetanilide drug, it has antipyretic and analgesic effect and is widely used in clinical practice. However, ACOP also has certain side effects to human, it is important to establish an approach for real-time detection of the ACOP content in human body. Currently, mainly detecting approaches are spectrophotometry, titration analysis, the first derivative spectroscopy, the high efficiency liquid chromatography and electrochemical methods $^{[4]}$ etc.. Using bare electrode for detecting drug molecules in electrochemistry is not a good choice, people usually pre-treat the working electrode used for detecting in order to improve the detection sensitivity, heated electrode technology has aroused widespread concern in the field of electrochemical analysis for its simple heating equipment,

\author{
Shaotong Jiang* \\ College of Biotechnology and Food Engineering, Hefei \\ University of Technology, Hefei, China,230009
}

higher detection sensitivity and lower electrode contamination effect ${ }^{[5]}$. However, restricted by heated electrode's production process, the working area of heated electrode is smaller, which impacts the detection effect, the use of nanomaterials especially two-dimensional plane carbon nanomaterial graphene for modifying heated electrode can significantly improve the heated electrode's plate electrode area, thus using it for drug molecules analysis is a very good idea. In this article, we first adopt the Hummers to prepare graphene oxide (GO), deoxygenize it to GR and then modify it in the heated glassy carbon electrode (HGCE). The GR modified HGCE has a great effect in detecting ACOP.

\section{TEST SECTION}

\section{A Reagents and Instruments}

CHI 660B electrochemical workstation (Shanghai Chinstrument Co., Ltd.); ECT-02 electrochemical electrode temperature modulation analyzer, the 213 platinum electrode, saturated calomel electrode, homemade heated glassy carbon electrode (HGCE).

Acetaminophen (ACOP), graphene (homemade); phosphate buffered saline (PBS) prepared with $\mathrm{Na}_{2} \mathrm{HPO}_{4}$ and $\mathrm{KH}_{2} \mathrm{PO}_{4}$ according to needs. Other chemical reagents are analytical reagents. Experimental water is double distilled water.

\section{B Preparation of Graphene}

Pre-oxidize the graphite referring the reported methods such as Kovtyukhova ${ }^{[6]}$ etc.: $3 \mathrm{~g}$ of graphite powder, $3.6 \mathrm{~g}$ of $\mathrm{K}_{2} \mathrm{~S}_{2} \mathrm{O}_{8}$ and $3.6 \mathrm{~g}$ of $\mathrm{P}_{2} \mathrm{O}_{5}$ are sequentially added to $12.0 \mathrm{ml}$ of concentrated sulfuric acid, continuously stir them for more than 6 hours under the condition of $80{ }^{\circ} \mathrm{C}$, dilute the mixture with $200 \mathrm{ml}$ of double distilled water then filter, wash and dry for preparing pre-oxidation of graphite powder.

Oxidize the above pre-oxidation of graphite to graphite oxide by using Hummers: add $0.5 \mathrm{~g}$ of homemade pre-oxidation of graphite to $23 \mathrm{ml}$ of concentrated sulfuric 
acid and stir for 2 hours under the condition of $0{ }^{\circ} \mathrm{C}$, in this process, slowly add $3.5 \mathrm{~g}$ of $\mathrm{KMnO}_{4}$ to the reaction system in six times, then heat the mixture to $35{ }^{\circ} \mathrm{C}$ and keep on reacting for 4 hours, keep on reacting for 40 minutes at $98^{\circ} \mathrm{C}$ after adding $46 \mathrm{ml}$ of deionized water, add $150 \mathrm{ml}$ of water to dilute it after cooling and add an appropriate amount of $30 \% \quad \mathrm{H}_{2} \mathrm{O}_{2}$ drop by drop, use $0.45 \mu \mathrm{m}$ microfiltration membrane for sucking filtration and use double distilled water to repeatedly wash until the filtrate's $\mathrm{pH}$ value is 5-6, dry it for 24 hours under the condition of $60{ }^{\circ} \mathrm{C}$ vacuum, then the graphene oxide $(\mathrm{GO})$ is finished.

Preparation of GR [ $\left.{ }^{8-10}\right]$ : add $0.1 \mathrm{~g}$ of graphene oxide to $100 \mathrm{ml}$ of double distilled water, use ultrasonic dissolution technique to peel it for 2 hours, add $0.5 \mathrm{ml}$ of $80 \%$ hydrazine hydrate solution, reflux it for 24 hours in $80^{\circ} \mathrm{Cbath}$, after cooling, use $0.45 \mu \mathrm{m}$ microfiltration membrane for sucking filtration, and then wash it, finally dry it for 24 hours under the condition of $50{ }^{\circ} \mathrm{C}$ vacuum, then the grphene (GR) is finished.

\section{Preparation of Modified Electrodes}

The preparation of HGCE is according to the literature ${ }^{[5]}$, take $0.3 \mathrm{~mm}$ glassy carbon electrode (GCE) as working electrode, wind the enameled wire near the bottom as heating wires by using double wound, the epoxy glue is used as working electrode after sealed and fixed, the underside of GCE is used as the working face of HGCE.

Polish the homemade HGCE's working face to mirror plane, wash it with double distilled water and adopt ultrasonic dissolution technique to wash it with 1: $1 \mathrm{HNO}$, ethanol and double distilled water for 3 minutes, dry it with Nitrogen; add $5 \mathrm{mg}$ of newly prepared GR to $10 \mathrm{ml}$ of $\mathrm{N}$ and N-Dimethylformamide solution and adopt ultrasonic dissolution technique to disperse it for 1 hour, therefore, the GR suspension is obtained. Measure appropriate amount of the above GR suspension by using the micro-injector, drop the suspension to the treated surface of HGCE, dry it with infrared light then the GR/HGCE is obtained. Place the dried modified electrode in the PBS buffer solution $(\mathrm{PH}=6.0)$ for activation.

\section{Experimental Methods}

Electrochemical detection adopts a three-electrode system, the graphene modified heated glassy carbon electrode (GR / HGCE) is used as working electrode, saturated calomel electrode as reference electrode, platinum electrode as counter electrode. $\mathrm{PH}=6.0 \mathrm{PBS}$ is used as supporting electrolyte. Scan the ACOP with differential pulse voltammetry in the range of $0.3-0.9 \mathrm{~V}$. Enrichment time: $150 \mathrm{~s}$, pulse amplitude: $50 \mathrm{mV}$, pulse width: $40 \mathrm{~ms}$, scan speed: $40 \mathrm{mV} / \mathrm{s}$. After each scan, place the electrode in blank base solution and repeatedly scan it until the peak disappears, then wash it with double distilled water, after drying by filter paper the next determination can be carried out.

\section{RESUlTS AND DiscUSSIONS}

\section{A Characterization of Homemade GR}

The X-ray powder diffraction pattern of Graphite (a) and GR (b) is shown in Figure 1, it can be concluded from Figure 1 (a) that in the untreated graphite's X-ray diffraction pattern, graphite has a sharp characteristic peak (at $26.5^{\circ}$ ), the presence of the peak proves the untreated graphite's structure is ordered crystalline. The GR generated after the oxidation is as shown in Figure 1(b): the characteristic peak at $26.5^{\circ}$ disappears, which indicates that the generated GR is a single structure. The ordered layered structure of graphite has been destroyed.

The infrared spectrum of GR (A) and Graphite (B) is shown in Figure 2, graphene has a distinct hydroxy peak (peak in Figure 2 (a)) at $3450 \mathrm{~cm}^{-1}$, which is $-\mathrm{OH}$ functional group in water, there is a distinct vibration's characteristic absorption peak ( peak in Figure 2 (b)) of carboxyl functional group $\mathrm{C}=\mathrm{C}$ at $1630 \mathrm{~cm}^{-1}$, and the infrared spectra of graphene at $1700 \mathrm{~cm}^{-1}$ represents the characteristic peak of stretching vibration peak when $\mathrm{C}=0$, accordingly it is considered that in the synthesis we have already deoxygenized the graphene oxide to graphene.

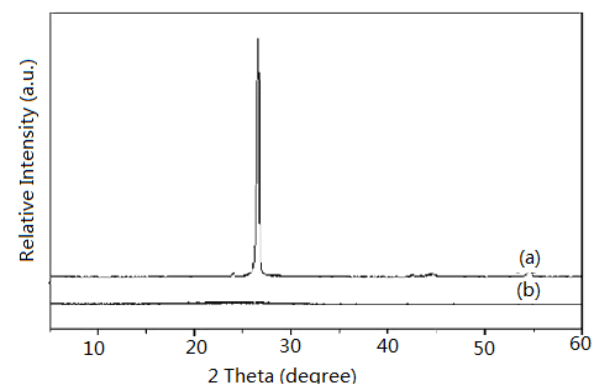

Figure 1. X-ray Powder Diffraction Pattern of Graphite (a) and GR (b)

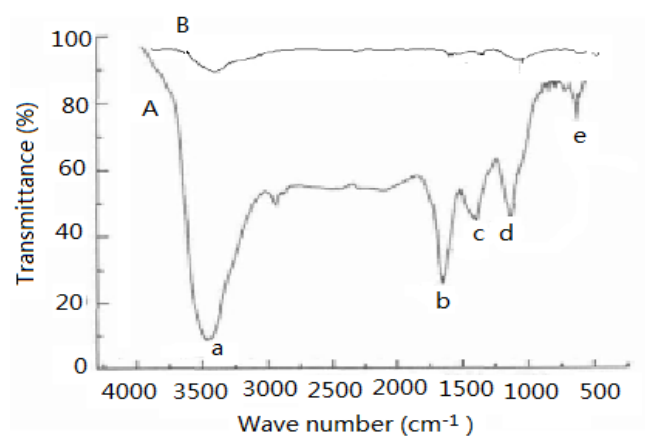

Figure 2. FI-IR Spectrum of Graphene(A) and Graphite ( B )

\section{B Characterization of Graphene Modified Heated Glassy} Carbon Electrode (GR / HGCE)

- Scanning Electron Microscope of GR / HGCE 
Use SEM to characterize GR/HGCE prepared in 1.2, as shown in Figure 3, the graphene on the surface of homemade graphene modified heated glassy carbon electrode contains fold structure and is disordered, parts of graphene is overlapped together to form a multilayer structure, the presence of the graphene layer greatly increases the effective area of glassy carbon electrode.

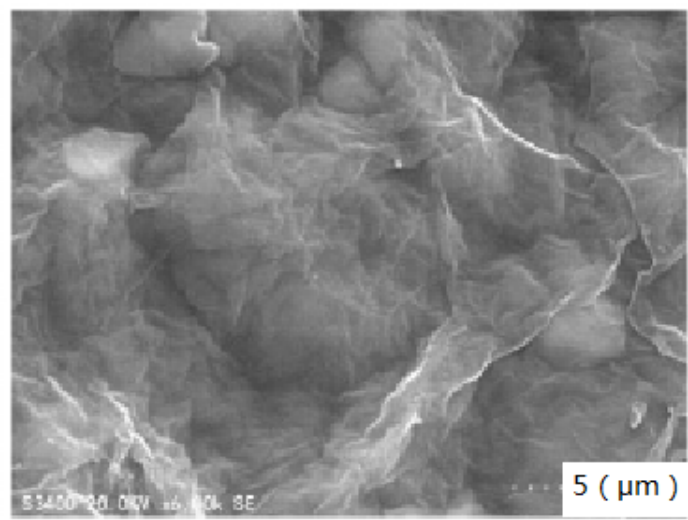

Figure 3. SEM image of GR/HGCE

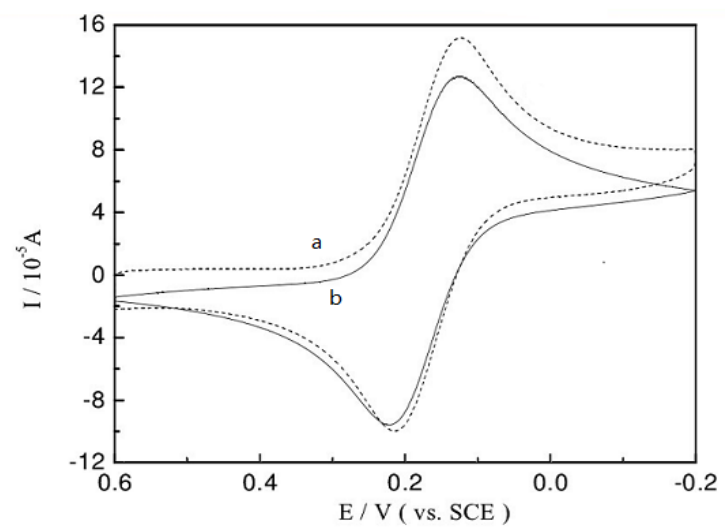

Figure 4. Cyclic Voltammogramss of $1 \times 10^{-2} \mathrm{~mol} / \mathrm{L}\left[\mathrm{Fe}(\mathrm{CN})_{6}\right]^{3-/ 4-}$ at $\mathrm{GCE}(\mathrm{b})$ and GR/HGCE(a)

- Electrochemical Performance Characterization of GR / HGCE

As shown in Figure 4, there is a significant increase in the cyclic volt-ampere current of the modified electrodes, in order to verify the modified electrode's electrochemical activity, the electrode's electrochemical impedance spectroscopy test ( the excitation amplitude is $5 \mathrm{mV}$, the frequency range is $1 \times 10^{-2}-1 \times 10^{4} \mathrm{~Hz}$, the additional constant potential is: $+200 \mathrm{mV}$ ) is carried out in this paper. EIS is a sensitive detection technology used to detect the impedance on electrode's surface in the modification process. EIS curve obtained from experiment can obtain resistance value $\left(R_{e t}\right)^{[11]}$ from the diameter of the semicircular portion in the high frequency region. The EIS of GCE or GR / HGCE are respectively investigated at room temperature, as shown in Figure 5, the semi-circular in high frequency region is corresponding to the charge transfer process in electrochemical reaction, the charge transfer resistance $R_{c t}$ can be directly obtained from the semi-circular's diameter. As can be seen from the figure, the impedance arc's radius of GR / HGCE is significantly smaller than that of GCE, which indicates its charge transfer resistance $\mathrm{R}_{\mathrm{ct}}$ significantly reduces. The mainly reasons that the resistance of graphite modified electrode significantly decreases is graphene itself has excellent heterogeneous electron transfer capability, which accelerates the electron transfer between $\left[\mathrm{Fe}(\mathrm{CN})_{6}\right]^{3-/ 4-}$ solution and the surface of electrode; on the other hand because of the existence of- $\mathrm{C}=$ $\mathrm{C}$ - inside the graphene's cardinal plane, which makes graphene poss a large $\pi$-conjugated system, accelerating the transfer of electrons in graphene's cardinal plane, so that the charge transfer resistance of GR / HGCE in $\left[\mathrm{Fe}(\mathrm{CN})_{6}\right]^{3-/ 4-}$ solution significantly reduces.

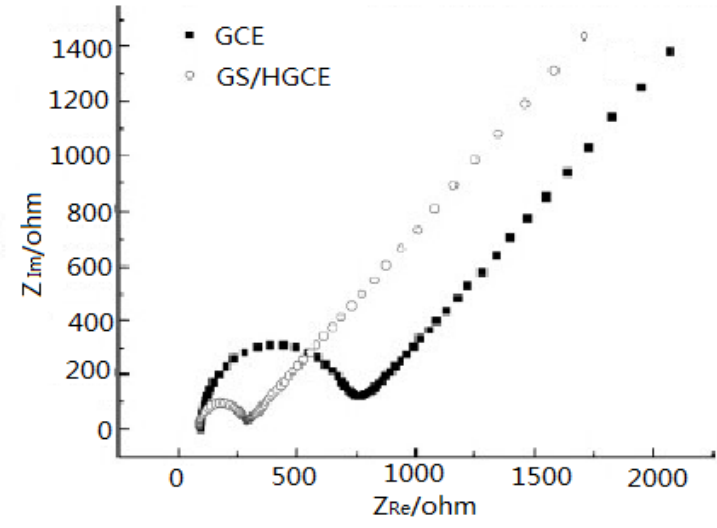

Figure 5. Nyquist of $\left[\mathrm{Fe}(\mathrm{CN})_{6}\right]^{3-/ 4-}$ on GCE and GR/HGCE

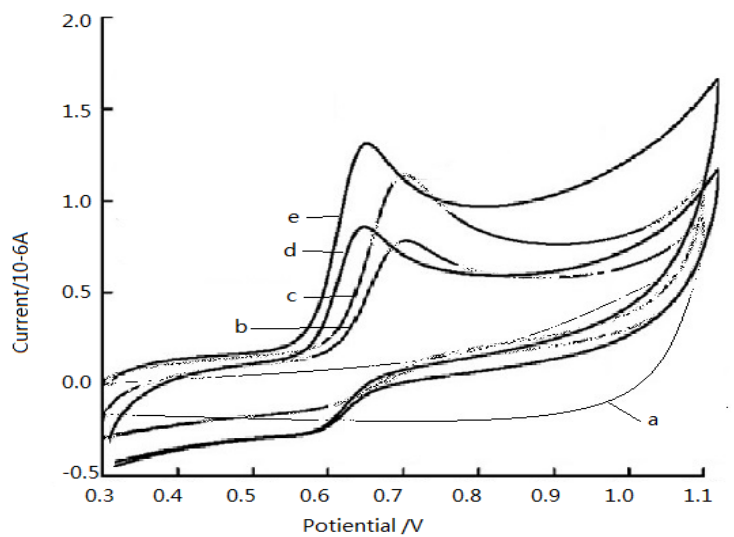

Figure 6. CV curves of ACOP on GCE、HGCE、GR/GCE and GR/HGCE in $\mathrm{PBS}(\mathrm{pH}=6.0)$. Scan Rate: $\mathrm{V}=100 \mathrm{mV} / \mathrm{s}$

- Cyclic Volt-ampere Diagram of ACOP on GR/HGCE

The electrochemical behavior of ACOP on the surface of GCE, HGCE, GR / GCE and GR / HGCE is studied by using cyclic voltammetry. As shown in Figure 6, C is ACOP's cyclic volt-ampere diagram on $\mathrm{HGCE}$, an oxidation peak $\mathrm{E}_{\mathrm{P} 2}$ appears at $0.689 \mathrm{~V}$, the peak height of $\mathrm{E}_{\mathrm{p} 2}$ significantly becomes larger compared to that of $\mathrm{Ep}_{1}$, which proves that 
the there is an increase in peak current, that is because the thermal effect of the heated electrode accelerates the electronic transfer rate on the electrode's surface, thus increasing the peak current; $d$ is ACOP's volt-ampere diagram on GR/GCE, an oxidation peak of ACOP $E_{P 3}$ appears near $0.640 \mathrm{~V}$, compared to $\mathrm{E}_{\mathrm{P} 1 \text {, its peak potential }}$ decreases and its peak current increases a little, a tiny reduction peak appears at $0.560 \mathrm{~V}$, which indicates that the presence of GR has a significant catalytic effect to the oxidation of ACOP, the electrons of ACOP on the modified membrane have a faster transfer rate; $\mathrm{e}$ is ACOP's volt-ampere diagram on GR/HGCE, there is also a oxidation peak $\mathrm{Ep}_{4}$ near $0.651 \mathrm{~V}$, its peak height is the highest, and its peak current obviously increases, that is because of the existence of GR, the significant catalysis effect of oxidation of GR coupled with the thermal effect of HGCE make electronic transfer rate faster and peak current highest.

\section{SELECtion of Test CONDitions}

\section{A Selection of the Amount of GR Modifier}

Verify the modified effect by changing the amount of GR solution, and the results show that in $0.5 \mathrm{mg} / \mathrm{ml}$ of graphene solution within the range of $0.1 \mathrm{uL}-10 \mathrm{uL}$, with the increase of modified liquid, the ACOP's peak current of oxidation peak on the modified electrode first becomes strong and then decreases, when dispensing amount reaches $6 \mathrm{uL}$, the peak current of ACOP reaches maximum. Therefore, the GR modified electrode prepared by $6 \mu \mathrm{L} \mathrm{mg} \mathrm{/}$ $\mathrm{mL}$ graphene suspension is adopted as working electrode in this paper.

\section{B Selection of Supporting Electrolyte}

Electrolyte and its $\mathrm{pH}$ value have an important impact on the peak potential and peak current of redox peaks of electro-active substances, the voltammetric behaviors of ACOP in hydrochloric acid, sulfuric acid, BR, NH3-NH4Cl, HAc-NaAc, PBS buffer solution of different concentrations and $\mathrm{pH}$ values are determined in this paper. The results show that the peak current reaches maximum, the peak shape is the best and the background current is relatively low when the $\mathrm{pH}$ value $=6.0 \mathrm{PBS}$ solutions is taken as the base solution. Therefore, the $\mathrm{pH}=6.0 \mathrm{PBS}$ is chosen as the supporting electrolyte.

\section{Effect of Enrichment Time}

Differential pulse voltammetry (DPV) has a higher sensitivity and resolution compared to cyclic voltammetry, therefore, here we select DPV to detect the electrochemical behaviors of different concentrations of ACOP. Investigate the DPV's enrichment potential and the open circuit's enrichment time before detection. The electrode's surface of $\mathrm{R} / \mathrm{HGCE}$ is modified by graphene owning a large effective surface area, and the graphene contains functional groups such as $\mathrm{C}=\mathrm{C}$ etc., so it has a high enrichment efficiency to ACOP, the detection signal changes with the increase of enrichment time, the oxidation peak's current of ACOP increases significantly when the enrichment time is within $0-240 \mathrm{~S}$, the oxidation peak's current of ACOP increases slowly when the enrichment time reaches $150 \mathrm{~S}$, which indicates that the concentration of ACOP on the surface of $\mathrm{GR} / \mathrm{HGCE}$ has tended to balance. So the enrichment time is chosen as 150 s.

\section{Effect of Electrode's Surface Temperature on Detection}

The electrochemical oxidation of ACOP on the surface of HGCE is also affected by the temperature of electrode. The temperature's effect is investigated under the above conditions. The heated electrode can significantly improve the electrochemical response signals, when the electrode is increasing in the range of $35-55^{\circ} \mathrm{C}$, the detecting current signal increasingly increases, however, when the temperature is $60{ }^{\circ} \mathrm{C}$, the inside of the electrolyte will generate bubbles, affecting the stability, so $55{ }^{\circ} \mathrm{C}$ is selected in this paper.

\section{E Precision, Reproducibility, Stability, Linearity Range and Interference Test}

The method's precision, reproducibility, stability and linearity range are investigated under the selected optimum conditions. The results show that use GR / HGCE to determine $20 \mu \mathrm{g} / \mathrm{ml}$ ACOP solution for 10 consecutive times, the RSD of peak current is $3.9 \%$, indicating that the electrode has good precision and reproducibility. The detecting linear range of ACOP is investigated by using DVP, the results indicate that the ACOP'S oxidation peak current $\left(i_{\text {pa }}, \mu \mathrm{A}\right.$ )on GR/HGCE has a good linear relationship with its concentration $(\mathrm{C}, \mu \mathrm{g} / \mathrm{mL})$ in the range of $0.04-20 \mathrm{ug} / \mathrm{mL}$, the regression equation is $i_{\mathrm{pa}}(\mu \mathrm{A})=$ $0.391 \mathrm{C}(\mu \mathrm{g} / \mathrm{mL})+1.18(\mathrm{r}=0.9966, \mathrm{n}=7)$, the detection limit is $13 \mu \mathrm{g} / \mathrm{L}\left(8.67 \times 10^{-8} \mathrm{~mol} / \mathrm{L}\right)$.

The interference experiment that how the common inorganic ions, organic compounds and pharmaceutical excipients impact methods is investigated under the selected optimum conditions, results show the detection's relative error is less than $\pm 5 \%, 50$ times of ascorbic acid, dopamine, fructose, cysteine, tyrosine, starch, 100 times of sucrose, glucose, mannitol, citric acid, etc. have no impact on the determination.

\section{F Determination and Standard Addition Recovery of ACOP in Samples}

Determine the amount of ACOP in different ACOP tablets and verify the standard addition recovery under the above optimal conditions. Respectively Fetch 2 ACOP tablets of six different brands for grinding, adopt ultrasonic dissolution technique to dissolve the tablets in ethanol, set the constant volume in $100 \mathrm{ml}$ flask and made into samples, when measuring, fetch appropriate amount of solution and diluted to 1000 times with the use of PBS buffer solution, conduct six times of parallel measurements, add the known amount of acetaminophen standard to the sample, determine it according to the same method, and then conduct the standard addition test, the standard addition recovery is between $98.9 \%$ to $103.5 \%$, RSD is $1.603 \%$. The test results are shown in Table 1. 
TABLE 1. DETERMINATION AND RECOVERY OF ACOP'S CONTENT IN TABLET

\begin{tabular}{|c|c|c|c|c|}
\hline $\begin{array}{c}\text { tagging } \\
\text { content } \\
(\mathrm{mg} / \mathrm{piece} \\
)\end{array}$ & $\begin{array}{c}\text { determined } \\
\text { values } \\
(\mathrm{mg} / \mathrm{piece} \\
)\end{array}$ & $\begin{array}{c}\text { added } \\
\text { amou } \\
\mathrm{nt} \\
(\mathrm{mg})\end{array}$ & $\begin{array}{c}\text { determin } \\
\text { ed } \\
\text { amount } \\
(\mathrm{mg})\end{array}$ & $\begin{array}{c}\text { recover } \\
\mathrm{y}\end{array}$ \\
\hline 100 & $98.42 \pm 1.52$ & 2 & 2.07 & 103.5 \\
\hline 100 & $97.28 \pm 0.82$ & 4 & 3.96 & 99.0 \\
\hline 300 & $295.3 \pm 3.19$ & 6 & 5.93 & 98.9 \\
\hline 300 & $297.5 \pm 2.17$ & 8 & 8.01 & 100.1 \\
\hline 500 & $497.3 \pm 2.64$ & 10 & 9.96 & 99.6 \\
\hline 500 & $498.4 \pm 3.17$ & 12 & 11.89 & 99.1 \\
\hline
\end{tabular}

\section{CONCLUSION}

The graphene is prepared in this paper. The graphene suspension is prepared to GR/HGCE by using dropping coating. Since the graphene has a special single atomic layer structure, it can significantly increase the effective surface area of HGCE; at the same, since the graphene has an catalytic effect to the electrochemical oxidation of ACOP, making the reactivity of ACOP on GR/HGCE significantly increases compared to that of GCE, GR/GCE, the electrochemical response signal enhances; the modified heated electrode can be applied to the actual sample analysis of ACOP.

\section{ACKNOWLEDGMENTS}

This work was supported by the research program of science at universities of Guangxi autonomous region (NO.
2013YB176) ,high levels of innovation team and excellence scholars program in colleges of Guangxi and foundation of guangxi university of science and technology (NO.1261115).

\section{REFERENCES}

[1] HUANG Yi, CHEN Yongsheng.Functionlization of Graphene and their Application[J].Science in China (Series B:Chemistry), , 2009,39(9): 887-896.

[2] HU Yao-Juan JIN Juan ZHANG Hui,etc.Acta Phys. -Chim. Sin., 2010, 26(8): 2073-2086.

[3] National Pharmacopoeia commission. National Drug Standard book[M].2002.236.

[4] LI Li-jun, CHENG Hao, CHEN Qi-feng,etal. Flow injection Irrevesible Biampermetric Determination of Acetaminophen [J].Journal of Instrumental Analysis,2006,25(5):38-40

[5] J Wang, M Jasinski, G U Flechsig, etal..Hot-wire amperometric monitoring of flowing streams. Talanta, 2000, 50(6):1205-1210.

[6] Kovtyukhova N I,Ollivier P J,Martin B R,etal.Layer- by -layer assembly of ultrathin composite films from micronsized graphite oxide sheets and polycations[J].Chem Mater,1999,11(3):771-778

[7] Hummers W S,Offeman R E.Preparation of graphitic oxide[J]. Am Chem Soc,1958,80(6):1339-1339

[8] Stankovich S, Dikin D A, Piner R D,etal. Synthesis of graphene based nanosheets via chemical reduction of exfoliated graphite oxide $[\mathrm{J}]$. Carbon, 2007, 45(7): 1558-1565 .

[9] Lomeda J R,Doyle C D,Kosynkin D V,etal. Diazonium functionalization of surfactant wrapped chemically converted graphene sheets[J].J Am Chem Soc , 2008 , 130(48):16201-16206 .

[10] Tung V C,Allen M J,Yang Y,etal.High-throughput solution processing of large-scale graphene[J]. Nature Nanotech , 2008 , 4(1):25-29.

[11] Guo H L, Wang X F, Qian Q Y, etal.A green approach to the synthesis of graphene nanosheets [J]. ACS Nano, 2009, 3 (9): 2653-2659. 